\title{
Mean Consumption, Poverty and Inequality in Rural India in the Sixtieth Round of the National Sample Survey
}

\author{
Raghbendra Jha, \\ Australian National \\ University
}

\author{
Raghav Gaiha, \\ University of Delhi
}

\author{
Anurag Sharma \\ Monash University
}

\begin{abstract}
This paper reports on mean consumption, poverty (all three FGT measures) and inequality during January to June 2004 for rural India using National sample Survey (NSS) data for the $60^{\text {th }}$ Round. Mean consumption at the national level is much higher than the poverty line. However, the Gini coefficient is higher than in recent earlier rounds. The headcount ratio using the thirty day recall is 22.9 per cent and with the seven day recall this stands at 17.9 per cent and, with the combined data, this figure is 20.6 per cent. Mean consumption, all three measures of poverty and the Gini coefficient are computed at the level of 20 states and 63 NSS regions in these 20 states. It is surmised that despite impressive growth rates deprivation is pervasive, pockets of severe poverty persist, and inequality is rampant.
\end{abstract}

All communication to:

Prof. Raghbendra Jha, Australia South Asia Research Centre, RSPAS, Australian National University Canberra, Act 0200

Australia

Phone: + 61261252683

Fax: + 61261250443

Email: r.jha@anu.edu.au 


\section{Mean Consumption, Poverty and Inequality in Rural India in the Sixtieth Round of the National Sample Survey}

\section{Introduction}

With the recent release of the National Sample Survey (NSS) data for the $60^{\text {th }}$ round (conducted during January to June 2004) the debate on the impact of the current economic

policy stance will, no doubt, be renewed anew. The change in the methodology for collecting NSS data put into effect in the $55^{\text {th }}$ Round (1999-00) created controversy about the comparability of the $55^{\text {th }}$ Round results with the earlier rounds.

The $60^{\text {th }}$ Round data is not entirely comparable with the $55^{\text {th }}$ Round data yet it does provide an inkling of how the movement of poverty has developed during this period. In this paper we provide estimates of mean consumption, poverty and inequality at three levels: the national level, state level and at the level of 63 NSS regions.

\section{Poverty During the 1990s}

A spate of studies (contained in Deaton and Kozel, 2005 and elsewhere) contain widely varying estimates of reduction in poverty during the 1990 s - a period that witnessed wideranging economic reforms — both domestic and external liberalisation — and accelerated growth. The reduction in poverty, however, varies depending on whether the estimates are obtained from adjusted or unadjusted $55^{\text {th }}$ round NSS estimates (see, for example, Sundaram and Tendulkar (2003, 2005), Deaton (2005), Datt, Kozel and Ravallion (2005), Deaton and Dreze (2002), Kijima and Lanjouw (2003) and Planning Commission, Government of India as reported in Sen and Himanshu (2004). To give the flavour of the debate, two sets of evidence are reviewed below. 
Although "official” estimates of poverty reduction are high (the HCR in rural areas fell from 37.27 per cent to 27.09 per cent, and the number of poor from 244 million to 193.2 million over the period 1993-99), the balance of evidence with careful adjustments of the 1999/2000 NSS data point to markedly lower reductions. An admirably comprehensive and by far the most persuasive is the contribution by Sen and Himanshu (2005). It casts doubts over earlier estimates of poverty reduction - specifically, that it was faster than that in the previous decade. As it turns out, the headcount ratio declined but at most by 3 percentage points and the absolute number of the poor did not decline over 1993-99. The reason is that both interstate and within-state inequality rose sharply during the 1990s, with the latter being the larger component of total inequality in India. Within-state urban inequality rose sharply in the 1990s, while a declining phase of within-state rural inequality was reversed during this period. So, despite rapid growth, poverty reduction in the 1990s was muted. The characterisation of the 1990s as "a relatively lost decade for poverty reduction" is thus not an exaggeration.

The reasons relate to weak linkages between liberalisation and agricultural growth on the one hand, and between the latter and poverty reduction on the other. First, although the growth rate of agricultural (crop) output was slightly higher during the 1990s (3.08 per cent annually as against 2.94 per cent during the 1980s), it slowed down to 1.6 per cent during 1997-98 and 2000/1. ${ }^{1}$ Secondly, whether agricultural growth translates into poverty reduction depends greatly on how unequal is the distribution of land. An implication of highly unequal distribution of land is that the growth of real agricultural wages tends to be sluggish. Independently of agricultural growth, abrupt increases in price of food result in higher

\footnotetext{
${ }^{1}$ For details, see Nayyar (2003).
} 
poverty. $^{2}$ Some additional but more recent evidence further points to slow reduction in poverty. (i) Between 1993-99, the employment elasticity of agricultural growth was barely 0.01 as compared to 0.70 during 1983-93. (ii) There was a sharp drop in the growth rate of real agricultural wages, from 3.99 per cent per annum during 1978-79 to 1989-90 to 2.34 per cent annually during 1990-91 to 1999-2000. (iii) The (wholesale) price index for food rose faster than that for all commodities. With $1980-81$ as the base at 100, food prices rose to 433 in 1998-99, while for all commodities it rose to only 353.3. ${ }^{3}$ In any case, the disadvantaged groups - especially the STs and female-headed households- continue to be considerably worse-off than all other rural households. ${ }^{4}$ Their social exclusion, and limited access to assets — especially land and education —.perpetuate their poverty (Gaiha and Imai, 2004).

Yet another dimension that needs critical scrutiny is spatial concentration of the poor. An analysis based on the NSS regional data over the period 1987-99 shows that the ranking of the poorest 20 regions has not changed much and that they continue to account for a large share of the rural poor. ${ }^{5}$ What these findings point to is that not only do persistently poor require greater access to human and physical assets, markets and financial services but also conditions that would enable them to overcome social exclusion. While a definitive comparison of the spatial concentration over the period covered by the earlier NSS rounds with the $60^{\text {th }}$ round must await a comparable regional classification, our analysis draws

\footnotetext{
${ }^{2}$ For illustrative results on the causal role of these variables based on the ICRISAT data for the semi-arid region, see Gaiha (1995).

${ }^{3}$ For details, see Nayyar (2003).

${ }^{4}$ In India, survey data for 1993-1994 show that per capita incomes among SCs were lower than the state averages (24 per cent in Andhra Pradesh, and 41 per cent in Kerala). In the eastern state of Orissa, more than 24 per cent of the population consisted of STs, as compared to 9 per cent in the country as a whole. 92 per cent of the households belonging to a ST in rural southern Orissa were poor - twice the state poverty rate and three and a half times the national poverty rate (CPRC, 2004). Besides, the overall prevalence of stunting among SC/ST children is much higher than the all-India average (over 63 per cent and about 58 per cent, respectively. For details, see Gaiha and Kulkarni (2005). See also Sundaram and Tendulkar (2005) for illustrations of relatively low rates of poverty reduction among subsets of SC/ST households during 1993-99.

${ }^{5}$ This is based on a collaborative study by R. Jha and R. Gaiha, funded by a DFID grant. See Jha and Gaiha (2003) and Jha and Sharma (2003).
} 
attention to marked variations within states and across regions as a continuing feature of poverty in India despite spectacular growth rates of income.

\section{Methodology}

The poverty measures used in this paper are all drawn from the popular Foster-GreerThorbecke (FGT) class of functions. This is written as:

$Y_{\alpha}=\sum_{y_{i}<z}\left[\left(z-y_{i}\right) / z\right]^{\alpha} / n$

where $Y$ is the measure of poverty, $y_{\mathrm{i}}$ is the (per capita) consumption expenditure of the $i$ th household or the ith class of household, $z$ is the poverty line, $n$ is the number of observations, and $\alpha$ is a non-negative parameter. The headcount ratio, PG0, given by the percentage of the population who are poor, is obtained when $\alpha=0$. This measure fails to capture the extent to which individual income (or expenditure) falls below the poverty line. Hence we use our second measure: the poverty gap index (PG1) given by the aggregate income shortfall of the poor as a proportion of the poverty line and normalized by the population size. This is given by setting $\alpha=1$ in (1). PG1 captures the acuteness of poverty since it measures the total shortfall of the poor from the poverty line. In other words, it measures the total amount of income necessary to remove that poverty. This measure has the drawback that it does not consider the importance of the number of people who are below the poverty line. For this reason, it is important to use both measures of poverty jointly to evaluate the extent of poverty. There are certain policy changes that favor one group of poor and adversely affect another group. In such cases PG0 may not register any change but PG1 may get around this problem to some extent. A further improvement is the Foster-Greer-Thorbecke measure which is obtained by setting $\alpha=2$ in (1). We abbreviate this as PG2. ${ }^{6}$

\footnotetext{
${ }^{6}$ This is also referred to as a distributionally sensitive measure. More generally, if $\alpha>\mathbf{1}$, the FGT index is distributionally sensitive.
} 
Also reported in this paper are data on the Gini coefficient, a standard measure of inequality. Typically this is defined as:

$G=\frac{\sum_{i=1}^{n} \sum_{j=1}^{n}\left|y_{i}-y_{j}\right|}{2 n^{2} \mu}$

Thus $G$ is calculated as the relative mean difference, i.e., the mean of the difference in consumption levels between every possible pair of households divided by the mean size $\mu$.

\section{Results}

Consumption data in the $60^{\text {th }}$ Round of the NSS has been reported in three formats:

(i) schedule 1 uses 30 day recall; (ii) schedule 2 uses 7 day recall; and (iii) consumption data combining the two recall periods are reported.

We present the results at three levels: (a) national; (b) state level (20 states) and (c) agroclimatic zone wise (63 NSS regions) for all three data formats. The poverty lines used in the analysis are reported in Table 1 . These poverty lines are updates for the official poverty lines used by the Government of India for the year 2004 using CPIAL figures for the nation as a whole (for India) and state level CPIALs.

Table 1: Poverty Lines Used in the Analysis: Rs. per capita per month

\begin{tabular}{llll}
\hline India & 399.00 & Assam & 406.65 \\
J\&K & 407.82 & West Bengal & 390.24 \\
Himachal Pradesh & 380.87 & Orissa & 375.00 \\
Punjab & 416.02 & Madhya Pradesh & 386.72 \\
Haryana & 420.71 & Gujarat & 410.16 \\
Rajasthan & 405.48 & Maharashtra & 410.15 \\
UP & 401.96 & Andhra Pradesh & 418.37 \\
Bihar & 379.69 & Karnataka & 398.45 \\
Manipur & 363.28 & Kerala & 411.34 \\
Tripura & 394.93 & Tamilnadu & 406.65 \\
Meghalaya & 421.88 & & \\
\hline
\end{tabular}


Computations on mean consumption, poverty and inequality at the national level are reported in Table 2.

Table 2: Mean Consumption, Poverty and Inequality in Rural India at the National Level

\begin{tabular}{|l|c|c|c|}
\hline & 30-Day recall & 7-day recall & Combined \\
\hline $\begin{array}{l}\text { Mean per capita consumption } \\
\text { (Rs. Per month) }\end{array}$ & 786 & 852 & 818 \\
\hline PG0 I & 22.90292 & 17.9421 & 20.62485 \\
\hline PG1 & 0.045277 & 0.034262 & 0.040367 \\
\hline PG2 & 0.01378 & 0.010126 & 0.012199 \\
\hline Gini Coefficient) & 0.36 & 0.37 & 0.37 \\
\hline
\end{tabular}

Although mean per capita consumption is much higher than the poverty line, the Gini coefficient is higher than in the earlier rounds. The Sen-Himanshu estimate (2005) for the $55^{\text {th }}$ round, for example, is 0.263 for the adjusted 7-day question on food. It may be noted, however, that our Gini estimates do not vary much with the recall period. But the poverty estimates do. These are lower with the 7-day recall. State level results for mean consumption are presented in Table 3.

\footnotetext{
${ }^{7}$ This and other mean consumption magnitudes are weighted means of expenditures.
} 
Table 3: Mean Consumption in Indian States (Rs. per capita per month)

\begin{tabular}{|c|c|c|c|}
\hline & 30-Day recall & 7-day recall & Combined \\
\hline $\mathrm{J} \& \mathrm{~K}$ & 972.8327 & 977.8441 & 968.4384 \\
\hline Himachal Pradesh & 973.8306 & 1144.433 & 1059.719 \\
\hline Punjab & 1083.278 & 1219.027 & 1152.276 \\
\hline Haryana & 982.1269 & 959.5434 & 969.3607 \\
\hline Rajasthan & 738.4412 & 772.5644 & 755.1089 \\
\hline UP & 689.8689 & 735.1858 & 708.3049 \\
\hline Bihar & 521.9517 & 546.1557 & 531.1039 \\
\hline Manipur & 723.9151 & 765.2466 & 739.9257 \\
\hline Tripura & 589.8687 & 658.7604 & 618.2768 \\
\hline Meghalaya & 788.2561 & 794.7582 & 789.8932 \\
\hline Assam & 640.9064 & 727.5854 & 685.3843 \\
\hline West Bengal & 800.8758 & 908.8001 & 856.5894 \\
\hline Orissa & 507.9771 & 606.8665 & 553.496 \\
\hline Madhya Pradesh & 570.1587 & 619.3557 & 595.461 \\
\hline Gujarat & 875.3434 & 941.0047 & 905.1426 \\
\hline Maharashtra & 975.7975 & 1088.055 & 1035.484 \\
\hline Andhra Pradesh & 788.5478 & 810.2635 & 801.5304 \\
\hline Karnataka & 717.1448 & 773.5267 & 744.5439 \\
\hline Kerala & 1220.356 & 1267.083 & 1242.271 \\
\hline Tamilnadu & 891.4005 & 990.3706 & 937.5418 \\
\hline
\end{tabular}

In Table 4 we report on state level poverty levels for all three FGT measures for all three recall measures. 
Table 4: State Level FGT Poverty measures

\begin{tabular}{|c|c|c|c|c|c|c|c|}
\hline & 30-day recall & 7-day recall & Combined & & 30-day recall & 7-day recall & Combined \\
\hline J\&K & & & & Assam & & & \\
\hline PG0 & 7.523135 & 3.666223 & 5.731677 & PG0 & 18.53069 & 9.585625 & 14.04115 \\
\hline PG1 & 0.009122 & 0.001207 & 0.005445 & PG1 & 0.032728 & 0.015354 & 0.024008 \\
\hline PG2 & 0.001685 & 4.57E-05 & 0.000924 & PG2 & 0.009242 & 0.00353 & 0.006375 \\
\hline Himachal Pradesh & & & & West Bengal & & & \\
\hline PG0 & 5.612861 & 4.884036 & 5.25185 & PGO & 15.64928 & 13.54053 & 14.64926 \\
\hline PG1 & 0.009318 & 0.006042 & 0.007695 & PG1 & 0.02817 & 0.020304 & 0.02444 \\
\hline PG2 & 0.002026 & 0.001058 & 0.001547 & PG2 & 0.008108 & 0.004658 & 0.006472 \\
\hline Punjab & & & & Orissa & & & \\
\hline PG0 & 4.345747 & 2.883431 & 3.627695 & PG0 & 48.976 & 34.07085 & 41.76606 \\
\hline PG1 & 0.006456 & 0.004189 & 0.005343 & PG1 & 0.121024 & 0.075288 & 0.098901 \\
\hline PG2 & 0.001769 & 0.000884 & 0.001334 & PG2 & 0.042643 & 0.025608 & 0.034402 \\
\hline Haryana & & & & Madhya Pradesh & & & \\
\hline PG0 & 4.745976 & 5.69111 & 5.207297 & PG0 & 34.72388 & 28.83626 & 31.84933 \\
\hline PG1 & 0.008585 & 0.00722 & 0.007919 & PG1 & 0.068514 & 0.059964 & 0.064339 \\
\hline PG2 & 0.002245 & 0.001726 & 0.001992 & PG2 & 0.01998 & 0.017173 & 0.01861 \\
\hline Rajasthan & & & & Gujarat & & & \\
\hline PG0 & 16.36508 & 12.84879 & 14.6321 & PGO & 16.52033 & 13.45861 & 14.99201 \\
\hline PG1 & 0.023992 & 0.018122 & 0.021099 & PG1 & 0.030296 & 0.017762 & 0.02404 \\
\hline PG2 & 0.005427 & 0.004975 & 0.005204 & PG2 & 0.007656 & 0.003812 & 0.005737 \\
\hline UP & & & & Maharashtra & & & \\
\hline PG0 & 27.45898 & 22.845 & 25.25927 & PG0 & 17.03622 & 14.00895 & 15.56448 \\
\hline PG1 & 0.052302 & 0.041244 & 0.04703 & PG1 & 0.028537 & 0.028206 & 0.028376 \\
\hline PG2 & 0.014608 & 0.011449 & 0.013102 & PG2 & 0.008707 & 0.008218 & 0.008469 \\
\hline Bihar & & & & Andhra Pradesh & & & \\
\hline PG0 & 36.56325 & 31.22599 & 33.98184 & PG0 & 27.66666 & 21.66952 & 24.71371 \\
\hline PG1 & 0.06722 & 0.054726 & 0.061177 & PG1 & 0.060019 & 0.042106 & 0.051199 \\
\hline PG2 & 0.018419 & 0.013893 & 0.01623 & PG2 & 0.019152 & 0.013391 & 0.016316 \\
\hline Manipur & & & & Karnataka & & & \\
\hline PG0 & 1.435798 & 0.348609 & 0.925893 & PGO & 26.43068 & 14.09506 & 20.49877 \\
\hline PG1 & 0.001101 & 0.000207 & 0.000682 & PG1 & 0.056255 & 0.028239 & 0.042783 \\
\hline PG2 & $9.41 \mathrm{E}-05$ & 1.34E-05 & $5.62 E-05$ & PG2 & 0.021097 & 0.010015 & 0.015768 \\
\hline Tripura & & & & Kerala & & & \\
\hline PGO & 25.31683 & 14.66559 & 20.24155 & PG0 & 6.465446 & 3.472056 & 5.004189 \\
\hline PG1 & 0.048087 & 0.021149 & 0.035251 & PG1 & 0.015452 & 0.005169 & 0.010432 \\
\hline PG2 & 0.012849 & 0.005601 & 0.009395 & PG2 & 0.005757 & 0.001017 & 0.003443 \\
\hline Meghalaya & & & & Tamilnadu & & & \\
\hline PG0 & 3.925776 & 3.756516 & 3.842222 & PG0 & 19.82947 & 15.11981 & 17.48075 \\
\hline PG1 & 0.001828 & 0.001995 & 0.00191 & PG1 & 0.037657 & 0.031844 & 0.034758 \\
\hline PG2 & 0.000197 & 0.000165 & 0.000181 & PG2 & 0.012383 & 0.010825 & 0.011606 \\
\hline
\end{tabular}


Some comments are in order. ${ }^{8}$

I. In all cases, the poverty estimates are lower with the weekly recall- in a few cases

(e.g. Assam, Orissa and Karnataka), the differences are large. This holds for all poverty indices.

II. Head count ratios vary considerably from 3.6 per cent for Punjab to 41.76 per cent for Orissa. Further there is considerable variation in the other two FGT measures of poverty. Thus there is great heterogeneity in poverty incidence across Indian states.

III. What is important to note is that some of the poorest states (viz. Bihar, UP, Orissa, Madhya Pradesh) continued to exhibit high incidence and severity of poverty in the $60^{\text {th }}$ round. As these states also accounted for a large majority of the poor, it is safe to conclude that large subsets of the rural population continue to remain in abject poverty.

IV. Among superior performers, Assam and Maharashtra stand out.

Turning now to within-state inequality we report on the Gini coefficients within the 20 Indian states for the three recall periods in Table 5.

Table 5: The Gini Coefficient in Select States: 2004

\begin{tabular}{|c|c|c|c|c|c|c|c|}
\hline State & $\begin{array}{c}\text { Gini } \\
\text { 30-day recall }\end{array}$ & $\begin{array}{c}\text { Gini } \\
\text { 7-day recall }\end{array}$ & $\begin{array}{c}\text { Gini } \\
\text { Combined }\end{array}$ & State & $\begin{array}{c}\text { Gini } \\
\text { 30-day recall }\end{array}$ & $\begin{array}{c}\text { Gini } \\
\text { 7-day recall }\end{array}$ & $\begin{array}{c}\text { Gini } \\
\text { Combined }\end{array}$ \\
\hline$J \& K$ & 0.2857 & 0.28686 & 0.2891 & Assam & 0.26354 & 0.25315 & 0.26268 \\
\hline Himachal Pradesh & 0.32247 & 0.34237 & 0.33768 & West Bengal & 0.35491 & 0.38784 & 0.37422 \\
\hline Punjab & 0.30412 & 0.34442 & 0.32985 & Orissa & 0.33115 & 0.35567 & 0.34649 \\
\hline Haryana & 0.28917 & 0.27829 & 0.2858 & Madhya Pradesh & 0.29297 & 0.31584 & 0.30892 \\
\hline Rajasthan & 0.29802 & 0.30682 & 0.30204 & Gujarat & 0.32668 & 0.3406 & 0.33397 \\
\hline UP & 0.34145 & 0.3431 & 0.3421 & Maharashtra & 0.38356 & 0.40212 & 0.39866 \\
\hline Bihar & 0.27494 & 0.26962 & 0.27377 & Andhra Pradesh & 0.39063 & 0.36082 & 0.37898 \\
\hline Manipur & 0.19766 & 0.1895 & 0.19364 & Karnataka & 0.3484 & 0.31728 & 0.33601 \\
\hline Tripura & 0.25951 & 0.24152 & 0.2518 & Kerala & 0.39086 & 0.34752 & 0.36902 \\
\hline Meghalaya & 0.24404 & 0.21329 & 0.22983 & Tamilnadu & 0.39833 & 0.40536 & 0.40275 \\
\hline
\end{tabular}

\footnotetext{
${ }^{8}$ Note that the comparisons are relative to the estimates given for the 30 day-recall with adjustments in Sen and Himanshu (2005), and essentially ordinal in nature.
} 
I. Apart from the relatively small north eastern states with low Ginis, in most other states the Ginis are high (e.g. Andhra Pradesh, Kerala, Tamil Nadu, Maharashtra, West Bengal, and UP0, pointing to considerable inequality in consumption expenditure. Other things being equal, and to the extent that consumption expenditure inequality reflects inequality in physical (e.g. land) and human capital (e.g. education), even high rates of growth of income are unlikely to translate into substantial poverty reduction. ${ }^{9}$

II. Our second observation is that the Gini and recall period are unrelated in so far as in some cases the 7-day recall Ginis are higher (e.g. Himachal Pradesh, Punjab, Madhya Pradesh, Gujarat, Maharashtra, West Bengal) while in others (e.g.Kerala, Karnataka, Andhra Pradesh, Haryana) the 30-day recall Ginis are higher.

We now report results for the 63 NSS regions in the 20 states studied in the sample. Since there is considerable heterogeneity in the mean income, poverty and inequality across Indian states we rank the NSS regions in respect of these criteria. Table 6 reports mean consumption across the 63 NSS regions.

\footnotetext{
${ }^{9}$ For poverty reduction, some forms of inequality matter more than others. Important ones include inequality in the distribution of assets, especially land, human capital, financial capital, and access to public assets, such as rural infrastructure. The fast growing economies of East and South East Asia had the advantage of low asset inequality compared to other Asian and Pacific economies — in some countries, following land reforms along with a better spread of education. Simulations for selected Asia countries show that even with low historic growth rates observed over 1980-98 continuing over the period 1998-2015, achievement of the MDG of halving poverty by 2015 will depend largely on reduction in income Gini. A case in point is Bangladesh. For details, see Gaiha et al. (2006).
} 
Table 6: Mean Consumption in 63 NSS regions.

\begin{tabular}{|c|c|c|c|c|c|}
\hline Region & $\begin{array}{c}\text { Mean per capita } \\
\text { consumption } \\
\text { Rs. per month, } \\
\text { using } 30 \text { day } \\
\text { recall }\end{array}$ & & $\begin{array}{c}\text { Mean per capita } \\
\text { consumption Rs. } \\
\text { per month, using } \\
7 \text { day recall }\end{array}$ & & $\begin{array}{c}\text { Mean per capita } \\
\text { consumption } \\
\text { Rs. per month, } \\
\text { using combined } \\
\text { data }\end{array}$ \\
\hline Orissa - Southern & 382.3202 & Bihar -Northern & 521.3547 & Orissa - Southern & 435.0203 \\
\hline Bihar - Northern & 493.9157 & Orissa-Southern & 531.7943 & Bihar - Northern & 506.5602 \\
\hline $\begin{array}{l}\text { Andhra Pradesh - } \\
\text { Southwestern }\end{array}$ & 497.7435 & Madhya Pradesh - Vindhya & 538.4066 & Madhya Pradesh - Vindhya & 534.5801 \\
\hline Madhya Pradesh - South & 513.1753 & Manipur Hills & 561.6731 & Manipur Hills & 542.8674 \\
\hline Manipur Hills & 529.0483 & Madhya Pradesh - Northern & 562.1582 & $\begin{array}{l}\text { Andhra Pradesh - } \\
\text { Southwestern }\end{array}$ & 545.6545 \\
\hline Orissa - Coastal & 533.5662 & Bihar -Central & 582.9039 & $\begin{array}{l}\text { Madhya Pradesh - } \\
\text { Southwestern }\end{array}$ & 566.5562 \\
\hline Madhya Pradesh - Vindhya & 538.9176 & Andhra Pradesh - Southwestern & 587.7381 & Bihar - Central & 567.6301 \\
\hline Orissa - Northern & 543.5959 & Assam- Hills & 588.5093 & Assam - Hills & 571.2477 \\
\hline $\begin{array}{l}\text { Madhya Pradesh - } \\
\text { Southwestern }\end{array}$ & 545.5946 & $\begin{array}{l}\text { Madhya Pradesh - } \\
\text { South Western }\end{array}$ & 591.7947 & Orissa - Coastal & 572.8435 \\
\hline Madhya Pradesh - Central & 547.9702 & Karnataka - Inland Northern & 603.7699 & Karnataka - Inland Northern & 582.4472 \\
\hline Assam - Hills & 563.392 & Orissa - Coastal & 609.3578 & Madhya Pradesh - South & 584.9811 \\
\hline Bihar - Central & 563.7171 & UP-Eastern & 620.5799 & Madhya Pradesh - Northern & 587.4471 \\
\hline $\begin{array}{l}\text { Karnataka - } \\
\text { Inland Northern }\end{array}$ & 564.5278 & J\&K - Outer Hills & 624.486 & Orissa - Northern & 591.7593 \\
\hline UP- Eastern & 574.0773 & UP - Southern & 632.0912 & UP - Eastern & 592.1753 \\
\hline $\begin{array}{l}\text { Maharashtra - } \\
\text { Inland Central }\end{array}$ & 585.1088 & Madhya Pradesh - South & 632.2123 & Madhya Pradesh - Central & 593.754 \\
\hline Rajasthan - Southern & 588.4957 & Rajasthan - Southern & 637.171 & Rajasthan - Southern & 611.4989 \\
\hline Tripura & 589.8687 & Madhya Pradesh - Central & 641.9581 & UP - Southern & 613.5037 \\
\hline Maharashtra- Eastern & 596.8489 & West Bengal - Eastern Plains & 642.4689 & Tripura & 618.2768 \\
\hline UP - Southern & 599.1825 & Orissa - Northern & 644.5159 & Maharashtra - Eastern & 635.4515 \\
\hline Rajasthan - Southeastern & 604.5262 & $\begin{array}{l}\text { Andhra Pradesh - } \\
\text { Inland Southern }\end{array}$ & 649.0862 & Maharashtra - Inland Central & 640.4635 \\
\hline $\begin{array}{l}\text { Madhya Pradesh - } \\
\text { Northern }\end{array}$ & 613.2689 & Tripura & 658.7604 & West Bengal - Eastern Plains & 640.8221 \\
\hline Assam - Western Plains & 626.8526 & Maharashtra - Eastern & 664.6354 & Assam - Western Plains & 663.589 \\
\hline $\begin{array}{l}\text { West Bengal - } \\
\text { Eastern Plains }\end{array}$ & 627.3531 & Assam - Western Plains & 696.3702 & West Bengal - Himalayan & 663.9409 \\
\hline $\begin{array}{l}\text { Maharashtra - } \\
\text { Inland Northern }\end{array}$ & 629.2679 & Maharashtra - Inland Central & 697.2817 & $\begin{array}{l}\text { Maharashtra - } \\
\text { Inland Northern }\end{array}$ & 670.1932 \\
\hline West Bengal - Himalayan & 633.6284 & West Bengal - Himalayan & 711.7695 & Madhya Pradesh - Malwa & 673.7985 \\
\hline Madhya Pradesh - Malwa & 640.1963 & Madhya Pradesh - Malwa & 712.6074 & $\begin{array}{l}\text { Andhra Pradesh - } \\
\text { Inland Southern }\end{array}$ & 677.9223 \\
\hline $\begin{array}{l}\text { West Bengal - Western } \\
\text { Plains }\end{array}$ & 646.5755 & Maharashtra-Inland Northern & 734.069 & J\&K - Outer Hills & 678.3652 \\
\hline Gujarat - Eastern Plains & 667.4835 & Gujarat-Eastern Plains & 745.4638 & Rajasthan - Southeastern & 688.3069 \\
\hline Assam - Eastern Plains & 670.2827 & Gujarat - Dry Areas & 751.0528 & $\begin{array}{l}\text { West Bengal - } \\
\text { Western Plains }\end{array}$ & 701.4953 \\
\hline Gujarat - Dry Areas & 677.9898 & West Bengal -Western Plains & 755.3273 & Gujarat - Eastern Plains & 707.1367 \\
\hline Karnataka - Inland Eastern & 697.7822 & Rajasthan-Southeastern & 773.8966 & Gujarat - Dry Areas & 710.1218 \\
\hline $\begin{array}{l}\text { Andhra Pradesh - } \\
\text { Inland Southern }\end{array}$ & 714.9236 & Assam- Eastern Plains & 789.0936 & Assam - Eastern Plains & 730.0946 \\
\hline $\begin{array}{l}\text { Maharashtra - } \\
\text { Inland Eastern }\end{array}$ & 721.8851 & Rajasthan-Western & 792.2087 & Karnataka - Inland Eastern & 762.0683 \\
\hline
\end{tabular}




\begin{tabular}{|c|c|c|c|c|c|}
\hline Tamilnadu - Southern & 722.0696 & Meghalaya & 794.7582 & Rajasthan - Western & 771.4897 \\
\hline UP - Central & 746.6434 & Rajasthan-Northeastern & 796.9272 & Andhra Pradesh - Coastal & 773.3236 \\
\hline Andhra Pradesh - Coastal & 747.1952 & Andhra Pradesh - Coastal & 800.4125 & Maharashtra - Inland Eastern & 778.3886 \\
\hline Rajasthan - Western & 749.8216 & UP -Western & 818.5796 & UP - Central & 780.0228 \\
\hline J\&K - Outer Hills & 767.5505 & UP -Central & 820.8091 & Meghalaya & 789.8932 \\
\hline Tamilnadu - Coastal & 782.2574 & Karnataka-Inland Eastern & 824.569 & UP - Western & 799.1729 \\
\hline $\begin{array}{l}\text { Karnataka - } \\
\text { Coastal and Ghats }\end{array}$ & 787.5728 & Haryana-Western & 841.1849 & Rajasthan - Northeastern & 804.0644 \\
\hline UP - Western & 787.6173 & Maharashtra-Inland Eastern & 850.0757 & Tamilnadu - Southern & 808.3885 \\
\hline Meghalaya & 788.2561 & Gujarat - Northern Plains & 863.3985 & $\begin{array}{l}\text { Karnataka - } \\
\text { Coastal and Ghats }\end{array}$ & 817.7058 \\
\hline Tamilnadu - Inland & 788.9409 & Karnataka-Coastal and Ghats & 864.4428 & Tamilnadu - Coastal & 821.3376 \\
\hline Rajasthan - Northeastern & 812.2178 & Tamilnadu-Coastal & 866.3769 & Haryana - Western & 828.0515 \\
\hline Manipur Plains & 822.3966 & Manipur Plains & 867.479 & Tamilnadu - Inland & 837.6813 \\
\hline Haryana - Western & 833.0341 & J\&K- Jhelum Valley & 882.9438 & Manipur Plains & 839.5699 \\
\hline J\&K - Jhelum Valley & 850.3121 & Tamilnadu-Southern & 892.0155 & J\&K - Jhelum Valley & 857.53 \\
\hline Gujarat - Northern Plains & 859.2667 & Tamilnadu-Inland & 902.9814 & Gujarat - Northern Plains & 862.1294 \\
\hline $\begin{array}{l}\text { Karnataka - } \\
\text { Inland Southern }\end{array}$ & 877.3021 & $\begin{array}{l}\text { Andhra Pradesh - } \\
\text { Inland Northern }\end{array}$ & 906.0837 & Karnataka - Inland Southern & 905.1707 \\
\hline $\begin{array}{l}\text { Andhra Pradesh - } \\
\text { Inland Northern }\end{array}$ & 917.9501 & Karnataka - Inland Southern & 930.2411 & $\begin{array}{l}\text { Andhra Pradesh - } \\
\text { Inland Northern }\end{array}$ & 918.4969 \\
\hline Kerala - Northern & 950.8168 & Kerala - Northern & 998.5377 & Kerala - Northern & 967.1696 \\
\hline Gujarat - Saurashtra & 964.5867 & Gujarat - Saurashtra & 999.3153 & Gujarat - Saurashtra & 973.1618 \\
\hline Punjab - Northern & 971.1683 & Haryana - Eastern & 1028.889 & Haryana - Eastern & 1052.442 \\
\hline Himachal Pradesh & 973.8306 & Maharashtra - Inland Western & 1103.052 & Himachal Pradesh & 1059.719 \\
\hline $\begin{array}{l}\text { West Bengal - } \\
\text { Central Plains }\end{array}$ & 987.8527 & Himachal Pradesh & 1144.433 & West Bengal - Central Plains & 1073.671 \\
\hline $\begin{array}{l}\text { Maharashtra - } \\
\text { Inland Western }\end{array}$ & 1051.11 & West Bengal - Central Plains & 1156.964 & $\begin{array}{l}\text { Maharashtra - } \\
\text { Inland Western }\end{array}$ & 1075.204 \\
\hline Haryana - Eastern & 1069.415 & Punjab - Northern & 1198.37 & Punjab - Northern & 1080.139 \\
\hline Gujarat - Southern Plains & 1091.632 & Tamilnadu - Coastal Northern & 1203.543 & Gujarat - Southern Plains & 1177.405 \\
\hline $\begin{array}{l}\text { Tamilnadu - } \\
\text { Coastal Northern }\end{array}$ & 1164.955 & Punjab - Southern & 1251.473 & $\begin{array}{l}\text { Tamilnadu - } \\
\text { Coastal Northern }\end{array}$ & 1180.791 \\
\hline Punjab - Southern & 1258.322 & Gujarat - Southern Plains & 1268.245 & Punjab - Southern & 1264.877 \\
\hline J\&K - Mountains & 1288.599 & J\&K - Mountains & 1364.163 & J\&K - Mountains & 1331.709 \\
\hline Kerala - Southern & 1370.839 & Kerala - Southern & 1417.252 & Kerala - Southern & 1396.197 \\
\hline Maharashtra - Coastal & 1504.582 & Maharashtra - Coastal & 1677.618 & Maharashtra - Coastal & 1614.205 \\
\hline
\end{tabular}

Tables 7, 8 and 9 give results on FGT measures of poverty for 30-day recall, 7-day recall and combined data for the 63 NSS regions. All figures have been arranged in ascending order to emphasise regional variations in the incidence and severity of poverty. 
Table 7: Poverty Incidence and severity in NSS regions: 30-day recall

\begin{tabular}{|c|c|c|c|c|c|}
\hline \multicolumn{6}{|l|}{30 - day recall } \\
\hline NSS Region & PGO & NSS Region & $P G 1$ & NSS Region & $P G 2$ \\
\hline Manipur Plains & 0 & Manipur Plains & 0 & Manipur Plains & 0 \\
\hline Haryana - Eastern & 1.995144 & Haryana - Eastern & 0.00179 & Meghalaya & 0.000199 \\
\hline J\&K - Mountains & 2.895294 & Meghalaya & 0.001845 & Haryana - Eastern & 0.000247 \\
\hline J\&K - Jhelum Valley & 2.907934 & Punjab - Northern & 0.003454 & Punjab - Northern & 0.000519 \\
\hline Maharashtra - Coastal & 3.634305 & Manipur Hills & 0.003498 & $\begin{array}{l}\text { Karnataka - } \\
\text { Coastal and Ghats }\end{array}$ & 0.00066 \\
\hline Meghalaya & 3.943499 & J\&K - Mountains & 0.004845 & J\&K - Jhelum Valley & 0.00114 \\
\hline Punjab - Northern & 3.9572 & Maharashtra - Coastal & 0.005388 & Maharashtra - Coastal & 0.001153 \\
\hline Kerala - Southern & 4.067028 & J\&K - Jhelum Valley & 0.005462 & J\&K - Mountains & 0.001246 \\
\hline Punjab - Southern & 4.1433 & $\begin{array}{l}\text { Karnataka - } \\
\text { Coastal and Ghats }\end{array}$ & 0.007159 & Himachal Pradesh & 0.001864 \\
\hline Manipur Hills & 4.561012 & Kerala - Southern & 0.007429 & J\&K - Outer Hills & 0.001942 \\
\hline Gujarat - Saurashtra & 5.228135 & Gujarat - Saurashtra & 0.008595 & Gujarat - Saurashtra & 0.001972 \\
\hline Himachal Pradesh & 5.345676 & Himachal Pradesh & 0.00864 & Kerala - Southern & 0.002225 \\
\hline Gujarat - Southern Plains & 6.689781 & Punjab - Southern & 0.010032 & $\begin{array}{l}\text { West Bengal - } \\
\text { Central Plains }\end{array}$ & 0.002374 \\
\hline Haryana - Western & 7.451478 & Gujarat - Southern Plains & 0.011498 & Gujarat - Southern Plains & 0.002509 \\
\hline $\begin{array}{l}\text { West Bengal - } \\
\text { Central Plains }\end{array}$ & 8.956159 & West Bengal - Central Plains & 0.011928 & Manipur Hills & 0.003498 \\
\hline Kerala - Northern & 10.92927 & J\&K - Outer Hills & 0.012849 & $\begin{array}{l}\text { Maharashtra - } \\
\text { Inland Western }\end{array}$ & 0.003785 \\
\hline $\begin{array}{l}\text { Maharashtra - Inland } \\
\text { Western }\end{array}$ & 13.23083 & Maharashtra - Inland Western & 0.014966 & $\begin{array}{l}\text { Rajasthan - } \\
\text { Northeastern }\end{array}$ & 0.003798 \\
\hline Rajasthan - Northeastern & 13.38379 & Karnataka - Inland Eastern & 0.015081 & Punjab - Southern & 0.003819 \\
\hline Rajasthan - Western & 14.01539 & Haryana - Western & 0.017723 & Rajasthan - Western & 0.004683 \\
\hline $\begin{array}{l}\text { Karnataka - } \\
\text { Coastal and Ghats }\end{array}$ & 14.03725 & Rajasthan - Northeastern & 0.018409 & Haryana - Western & 0.005066 \\
\hline Gujarat - Northern Plains & 16.34344 & Rajasthan - Western & 0.021642 & Assam - Eastern Plains & 0.005972 \\
\hline Rajasthan - Southeastern & 16.4631 & UP - Western & 0.024194 & UP - Western & 0.006121 \\
\hline UP - Western & 16.57037 & Assam - Eastern Plains & 0.025918 & Rajasthan - Southern & 0.006542 \\
\hline J\&K - Outer Hills & 17.4346 & Gujarat - Northern Plains & 0.026913 & Gujarat - Northern Plains & 0.006549 \\
\hline Tamilnadu - Southern & 17.5686 & Tamilnadu - Southern & 0.029134 & Tamilnadu - Southern & 0.00734 \\
\hline Assam - Eastern Plains & 17.64185 & Tamilnadu - Inland & 0.029689 & Tamilnadu - Inland & 0.007541 \\
\hline Tamilnadu - Inland & 18.15908 & Kerala - Northern & 0.03021 & $\begin{array}{l}\text { West Bengal - } \\
\text { Western Plains }\end{array}$ & 0.009512 \\
\hline Assam - Western Plains & 18.40118 & West Bengal - Western Plains & 0.032159 & $\begin{array}{l}\text { Maharashtra - } \\
\text { Inland Eastern }\end{array}$ & 0.010248 \\
\hline Karnataka - Inland Southern & 19.6512 & Maharashtra - Inland Eastern & 0.033541 & $\begin{array}{l}\text { Madhya Pradesh - } \\
\text { Southwestern }\end{array}$ & 0.01051 \\
\hline $\begin{array}{l}\text { West Bengal - } \\
\text { Western Plains }\end{array}$ & 20.13886 & Assam - Western Plains & 0.035572 & Assam - Western Plains & 0.011132 \\
\hline $\begin{array}{l}\text { Tamilnadu - } \\
\text { Coastal Northern }\end{array}$ & 20.25069 & Rajasthan - Southeastern & 0.038519 & UP - Southern & 0.011806 \\
\hline $\begin{array}{l}\text { Maharashtra - } \\
\text { Inland Northern }\end{array}$ & 20.52224 & Rajasthan - Southern & 0.03967 & Gujarat - Eastern Plains & 0.011842 \\
\hline $\begin{array}{l}\text { West Bengal - } \\
\text { Eastern Plains }\end{array}$ & 20.71352 & Tamilnadu - Coastal Northern & 0.040416 & Kerala - Northern & 0.01211 \\
\hline
\end{tabular}


Mean Consumption, Poverty \& Inequality in Rural India in the $60^{\text {th }}$ Round of the NSS

\begin{tabular}{|c|c|c|c|c|c|}
\hline $\begin{array}{l}\text { Maharashtra - Inland } \\
\text { Eastern }\end{array}$ & 21.1517 & West Bengal - Eastern Plains & 0.04364 & $\begin{array}{l}\text { Andhra Pradesh - } \\
\text { Coastal }\end{array}$ & 0.012265 \\
\hline Andhra Pradesh - Coastal & 23.32787 & $\begin{array}{l}\text { Madhya Pradesh - } \\
\text { Southwestern }\end{array}$ & 0.044417 & $\begin{array}{l}\text { Andhra Pradesh - } \\
\text { Inland Northern }\end{array}$ & 0.012409 \\
\hline Tamilnadu - Coastal & 23.38454 & Andhra Pradesh - Coastal & 0.044961 & Tripura & 0.012554 \\
\hline UP - Central & 24.17432 & UP - Southern & 0.045832 & $\begin{array}{l}\text { Rajasthan - } \\
\text { Southeastern }\end{array}$ & 0.013439 \\
\hline Maharashtra - Eastern & 24.2277 & $\begin{array}{l}\text { Maharashtra - } \\
\text { Inland Northern }\end{array}$ & 0.04638 & Madhya Pradesh - Malwa & 0.01359 \\
\hline Tripura & 24.34617 & Tripura & 0.046556 & UP - Central & 0.013888 \\
\hline $\begin{array}{l}\text { Andhra Pradesh - } \\
\text { Inland Southern }\end{array}$ & 24.54459 & $\begin{array}{l}\text { Andhra Pradesh - } \\
\text { Inland Northern }\end{array}$ & 0.046842 & $\begin{array}{l}\text { West Bengal - Eastern } \\
\text { Plains }\end{array}$ & 0.014368 \\
\hline Karnataka - Inland Eastern & 25.49373 & Assam - Hills & 0.046902 & $\begin{array}{l}\text { Madhya Pradesh - } \\
\text { Northern }\end{array}$ & 0.014505 \\
\hline Assam - Hills & 25.53743 & Madhya Pradesh - Malwa & 0.047673 & Assam - Hills & 0.014948 \\
\hline $\begin{array}{l}\text { Andhra Pradesh - } \\
\text { Inland Northern }\end{array}$ & 25.89149 & UP - Central & 0.048833 & Bihar - Northern & 0.015079 \\
\hline UP - Southern & 26.07072 & Tamilnadu - Coastal & 0.05029 & $\begin{array}{l}\text { Tamilnadu - Coastal } \\
\text { Northern }\end{array}$ & 0.015404 \\
\hline Madhya Pradesh - Malwa & 27.08645 & Karnataka - Inland Southern & 0.054795 & $\begin{array}{l}\text { Maharashtra - Inland } \\
\text { Central }\end{array}$ & 0.015429 \\
\hline Gujarat - Dry Areas & 28.54501 & Madhya Pradesh - Northern & 0.05722 & West Bengal - Himalayan & 0.018159 \\
\hline Madhya Pradesh - Vindhya & 28.67239 & Gujarat - Eastern Plains & 0.058257 & Tamilnadu - Coastal & 0.018276 \\
\hline $\begin{array}{l}\text { Madhya Pradesh - } \\
\text { Southwestern }\end{array}$ & 29.64371 & Maharashtra - Inland Central & 0.060293 & $\begin{array}{l}\text { Karnataka - } \\
\text { Inland Northern }\end{array}$ & 0.01844 \\
\hline West Bengal - Himalayan & 29.84214 & Bihar - Northern & 0.0612 & Gujarat - Dry Areas & 0.019964 \\
\hline Karnataka - Inland Northern & 33.87849 & Madhya Pradesh - Vindhya & 0.061715 & Orissa - Coastal & 0.019994 \\
\hline $\begin{array}{l}\text { Maharashtra - } \\
\text { Inland Central }\end{array}$ & 34.40388 & Gujarat - Dry Areas & 0.062646 & $\begin{array}{l}\text { Madhya Pradesh - } \\
\text { Vindhya }\end{array}$ & 0.020289 \\
\hline Gujarat - Eastern Plains & 34.68014 & Maharashtra - Eastern & 0.063384 & $\begin{array}{l}\text { Maharashtra - } \\
\text { Inland Northern }\end{array}$ & 0.020336 \\
\hline Rajasthan - Southern & 35.32853 & West Bengal - Himalayan & 0.064137 & Maharashtra - Eastern & 0.021033 \\
\hline Bihar - Central & 35.55847 & $\begin{array}{l}\text { Andhra Pradesh - } \\
\text { Inland Southern }\end{array}$ & 0.065284 & Bihar - Central & 0.021051 \\
\hline Madhya Pradesh - Northern & 35.59961 & Karnataka - Inland Northern & 0.06628 & $\begin{array}{l}\text { Madhya Pradesh - } \\
\text { Central }\end{array}$ & 0.023015 \\
\hline Bihar - Northern & 35.91731 & Orissa - Coastal & 0.069735 & $\begin{array}{l}\text { Andhra Pradesh - } \\
\text { Inland Southern }\end{array}$ & 0.023662 \\
\hline Orissa - Coastal & 37.76379 & Bihar - Central & 0.07055 & $\begin{array}{l}\text { Karnataka - Inland } \\
\text { Eastern }\end{array}$ & 0.023662 \\
\hline UP - Eastern & 39.34115 & Madhya Pradesh - Central & 0.081684 & UP - Eastern & 0.023787 \\
\hline Madhya Pradesh - Central & 41.56143 & UP - Eastern & 0.081875 & $\begin{array}{l}\text { Karnataka - } \\
\text { Inland Southern }\end{array}$ & 0.025383 \\
\hline Madhya Pradesh - South & 44.71255 & Madhya Pradesh - South & 0.107487 & Madhya Pradesh - South & 0.033419 \\
\hline Orissa - Northern & 48.74789 & Orissa - Northern & 0.126256 & Orissa - Northern & 0.046679 \\
\hline $\begin{array}{l}\text { Andhra Pradesh - } \\
\text { Southwestern }\end{array}$ & 63.49303 & $\begin{array}{l}\text { Andhra Pradesh - } \\
\text { Southwestern }\end{array}$ & 0.197167 & $\begin{array}{l}\text { Andhra Pradesh - } \\
\text { Southwestern }\end{array}$ & 0.080099 \\
\hline Orissa - Southern & 71.9851 & Orissa - Southern & 0.227887 & Orissa - Southern & 0.08701 \\
\hline
\end{tabular}


Table 8: Poverty Incidence and Severity in NSS regions: 7-day recall

\begin{tabular}{|c|c|c|c|c|c|}
\hline 7 - day recall & PGO & & PG1 & & PG2 \\
\hline$J \& K$ - Mountains & 0 & J\&K - Mountains & 0 & J\&K - Mountains & 0 \\
\hline Manipur Plains & 0 & Manipur Plains & 0 & Manipur Plains & 0 \\
\hline $\begin{array}{l}\text { Karnataka - } \\
\text { Coastal and Ghats }\end{array}$ & 0 & $\begin{array}{l}\text { Karnataka - } \\
\text { Coastal and Ghats }\end{array}$ & 0 & $\begin{array}{l}\text { Karnataka - } \\
\text { Coastal and Ghats }\end{array}$ & 0 \\
\hline Manipur Hills & 0.97492 & J\&K - Jhelum Valley & $8.32 E-05$ & J\&K - Jhelum Valley & 4.86E - 07 \\
\hline Karnataka - Inland Eastern & 1.333028 & Manipur Hills & 0.000578 & Manipur Hills & 3.74E - 05 \\
\hline J\&K - Jhelum Valley & 1.424487 & Karnataka - Inland Eastern & 0.000729 & $\begin{array}{l}\text { Karnataka - } \\
\text { Inland Eastern }\end{array}$ & $3.98 \mathrm{E}-05$ \\
\hline Punjab - Northern & 2.065513 & Haryana - Eastern & 0.001386 & Haryana - Eastern & $9.87 \mathrm{E}-05$ \\
\hline Maharashtra - Coastal & 2.411363 & Punjab - Northern & 0.001897 & J\&K - Outer Hills & 0.000167 \\
\hline Haryana - Eastern & 2.842309 & Meghalaya & 0.002024 & Punjab - Northern & 0.000174 \\
\hline Kerala - Southern & 3.254454 & Assam - Hills & 0.002745 & Meghalaya & 0.000174 \\
\hline Gujarat - Saurashtra & 3.510835 & Maharashtra - Coastal & 0.003524 & Assam - Hills & 0.000251 \\
\hline Meghalaya & 3.748284 & Kerala - Southern & 0.004053 & Maharashtra - Coastal & 0.000736 \\
\hline Punjab - Southern & 4.01827 & J\&K - Outer Hills & 0.004284 & Assam - Eastern Plains & 0.000742 \\
\hline Kerala - Northern & 4.387776 & Assam - Eastern Plains & 0.005404 & Kerala - Southern & 0.000825 \\
\hline Assam - Hills & 4.561741 & Himachal Pradesh & 0.005858 & Himachal Pradesh & 0.001026 \\
\hline Himachal Pradesh & 4.795449 & Punjab - Southern & 0.007226 & Kerala - Northern & 0.001285 \\
\hline Assam - Eastern Plains & 6.350944 & Kerala - Northern & 0.007229 & Gujarat - Northern Plains & 0.001519 \\
\hline West Bengal - Central Plains & 6.484249 & Gujarat - Saurashtra & 0.007927 & Punjab - Southern & 0.001783 \\
\hline Karnataka - Inland Southern & 7.923218 & West Bengal - Central Plains & 0.009405 & $\begin{array}{l}\text { Madhya Pradesh - } \\
\text { Southwestern }\end{array}$ & 0.001954 \\
\hline Gujarat - Southern Plains & 8.775191 & Rajasthan - Northeastern & 0.012517 & $\begin{array}{l}\text { West Bengal - } \\
\text { Central Plains }\end{array}$ & 0.002118 \\
\hline Tamilnadu - Inland & 9.206844 & $\begin{array}{l}\text { Madhya Pradesh - } \\
\text { Southwestern }\end{array}$ & 0.012762 & Rajasthan - Northeastern & 0.002674 \\
\hline Rajasthan - Western & 9.948625 & Gujarat - Northern Plains & 0.014159 & Gujarat - Saurashtra & 0.002875 \\
\hline $\begin{array}{l}\text { Maharashtra - } \\
\text { Inland Western }\end{array}$ & 9.981079 & $\begin{array}{l}\text { West Bengal - } \\
\text { Western Plains }\end{array}$ & 0.015367 & Tamilnadu - Coastal & 0.002955 \\
\hline Haryana - Western & 10.52494 & Maharashtra - Inland Western & 0.015481 & $\begin{array}{l}\text { West Bengal - } \\
\text { Western Plains }\end{array}$ & 0.003024 \\
\hline $\begin{array}{l}\text { Madhya Pradesh - } \\
\text { Southwestern }\end{array}$ & 10.66594 & Haryana - Western & 0.016243 & $\begin{array}{l}\text { Maharashtra - Inland } \\
\text { Western }\end{array}$ & 0.003718 \\
\hline J\&K - Outer Hills & 11.01397 & Tamilnadu - Coastal & 0.01641 & Gujarat - Dry Areas & 0.004049 \\
\hline UP - Western & 11.84736 & Rajasthan - Western & 0.016589 & Haryana - Western & 0.004199 \\
\hline Rajasthan - Northeastern & 13.03963 & Tamilnadu - Inland & 0.017995 & UP - Western & 0.004266 \\
\hline Assam - Western Plains & 13.15418 & UP - Western & 0.018813 & Rajasthan - Western & 0.004583 \\
\hline Tamilnadu - Coastal & 13.7651 & Karnataka - Inland Southern & 0.021252 & Tamilnadu - Inland & 0.005001 \\
\hline $\begin{array}{l}\text { Maharashtra - Inland } \\
\text { Northern }\end{array}$ & 13.94992 & Tripura & 0.021659 & Gujarat - Eastern Plains & 0.005184 \\
\hline $\begin{array}{l}\text { West Bengal - } \\
\text { Western Plains }\end{array}$ & 14.3031 & Gujarat - Dry Areas & 0.02245 & Tripura & 0.005615 \\
\hline Tripura & 14.81768 & Gujarat - Southern Plains & 0.023258 & Assam - Western Plains & 0.006164 \\
\hline Rajasthan - Southeastern & 15.32071 & Assam - Western Plains & 0.024826 & $\begin{array}{l}\text { Andhra Pradesh - } \\
\text { Inland Northern }\end{array}$ & 0.006918 \\
\hline Andhra Pradesh - Coastal & 15.68051 & $\begin{array}{l}\text { Maharashtra - } \\
\text { Inland Northern }\end{array}$ & 0.027101 & Gujarat - Southern Plains & 0.006944 \\
\hline Tamilnadu - Southern & 16.3625 & Rajasthan - Southern & 0.027602 & Rajasthan - Southern & 0.006958 \\
\hline
\end{tabular}




\begin{tabular}{|c|c|c|c|c|c|}
\hline Gujarat - Dry Areas & 16.67275 & Gujarat - Eastern Plains & 0.028074 & $\begin{array}{l}\text { Maharashtra - } \\
\text { Inland Northern }\end{array}$ & 0.007256 \\
\hline Maharashtra - Inland Eastern & 17.8731 & $\begin{array}{l}\text { Andhra Pradesh - } \\
\text { Inland Northern }\end{array}$ & 0.030056 & $\begin{array}{l}\text { Karnataka - } \\
\text { Inland Southern }\end{array}$ & 0.008222 \\
\hline Gujarat - Northern Plains & 17.9742 & Andhra Pradesh - Coastal & 0.030489 & $\begin{array}{l}\text { West Bengal - } \\
\text { Eastern Plains }\end{array}$ & 0.008967 \\
\hline Rajasthan - Southern & 19.19664 & Rajasthan - Southeastern & 0.033054 & West Bengal - Himalayan & 0.009032 \\
\hline Tamilnadu - Coastal Northern & 19.79076 & UP - Southern & 0.033531 & UP - Southern & 0.009632 \\
\hline $\begin{array}{l}\text { Andhra Pradesh - } \\
\text { Inland Northern }\end{array}$ & 20.83763 & Maharashtra - Inland Eastern & 0.035372 & Orissa - Coastal & 0.009961 \\
\hline Madhya Pradesh - Malwa & 20.91897 & Orissa - Coastal & 0.035994 & Andhra Pradesh - Coastal & 0.009995 \\
\hline West Bengal - Eastern Plains & 20.91946 & West Bengal - Eastern Plains & 0.03631 & $\begin{array}{l}\text { Maharashtra - } \\
\text { Inland Eastern }\end{array}$ & 0.010605 \\
\hline UP - Southern & 22.05139 & UP - Central & 0.03904 & UP - Central & 0.011241 \\
\hline Gujarat - Eastern Plains & 22.83245 & West Bengal - Himalayan & 0.039401 & Rajasthan - Southeastern & 0.011955 \\
\hline UP - Central & 23.51086 & Tamilnadu - Southern & 0.041147 & Bihar - Central & 0.012205 \\
\hline Orissa - Coastal & 23.86789 & Tamilnadu - Coastal Northern & 0.043193 & $\begin{array}{l}\text { Madhya Pradesh - } \\
\text { Northern }\end{array}$ & 0.012713 \\
\hline Maharashtra - Inland Central & 25.47781 & Madhya Pradesh - Malwa & 0.046021 & Madhya Pradesh - Malwa & 0.013683 \\
\hline Karnataka - Inland Northern & 25.85852 & Karnataka - Inland Northern & 0.046657 & Tamilnadu - Southern & 0.0139 \\
\hline West Bengal - Himalayan & 25.9625 & Bihar - Central & 0.047977 & Bihar - Northern & 0.014546 \\
\hline Bihar - Central & 28.43318 & Maharashtra - Inland Central & 0.056167 & $\begin{array}{l}\text { Karnataka - } \\
\text { Inland Northern }\end{array}$ & 0.015972 \\
\hline $\begin{array}{l}\text { Andhra Pradesh - Inland } \\
\text { Southern }\end{array}$ & 29.36675 & Bihar - Northern & 0.057812 & $\begin{array}{l}\text { Tamilnadu - } \\
\text { Coastal Northern }\end{array}$ & 0.01689 \\
\hline Madhya Pradesh - Central & 29.37299 & Madhya Pradesh - Northern & 0.059338 & $\begin{array}{l}\text { Madhya Pradesh - } \\
\text { Vindhya }\end{array}$ & 0.017835 \\
\hline Bihar - Northern & 32.6473 & UP - Eastern & 0.065587 & $\begin{array}{l}\text { Maharashtra - } \\
\text { Inland Central }\end{array}$ & 0.018612 \\
\hline Madhya Pradesh - Northern & 32.90439 & Madhya Pradesh - Vindhya & 0.067507 & UP - Eastern & 0.019237 \\
\hline UP - Eastern & 33.20043 & Madhya Pradesh - Central & 0.067988 & $\begin{array}{l}\text { Madhya Pradesh - } \\
\text { Central }\end{array}$ & 0.02113 \\
\hline Madhya Pradesh - Vindhya & 34.75217 & $\begin{array}{l}\text { Andhra Pradesh - } \\
\text { Inland Southern }\end{array}$ & 0.069988 & $\begin{array}{l}\text { Andhra Pradesh - } \\
\text { Inland Southern }\end{array}$ & 0.023986 \\
\hline Madhya Pradesh - South & 38.1685 & Madhya Pradesh - South & 0.088487 & Maharashtra - Eastern & 0.027819 \\
\hline Maharashtra - Eastern & 39.01457 & Maharashtra - Eastern & 0.093848 & Madhya Pradesh - South & 0.029234 \\
\hline Orissa - Southern & 39.51829 & Orissa - Southern & 0.103585 & Orissa - Southern & 0.036849 \\
\hline Orissa - Northern & 42.80474 & Orissa - Northern & 0.108525 & Orissa - Northern & 0.039421 \\
\hline $\begin{array}{l}\text { Andhra Pradesh - } \\
\text { Southwestern }\end{array}$ & 45.428 & $\begin{array}{l}\text { Andhra Pradesh - } \\
\text { Southwestern }\end{array}$ & 0.124597 & $\begin{array}{l}\text { Andhra Pradesh - } \\
\text { Southwestern }\end{array}$ & 0.045835 \\
\hline
\end{tabular}


Table 9: Poverty Incidence and severity in NSS regions: Combined data

\begin{tabular}{|c|c|c|c|c|c|}
\hline \multicolumn{6}{|l|}{ Combined } \\
\hline NSS Region & PGO & NSS Region & PG1 & NSS Region & PG2 \\
\hline Manipur Plains & 0 & Manipur Plains & 0 & Manipur Plains & 0 \\
\hline J\&K - Mountains & 1.445786 & Haryana - Eastern & 0.001588 & Manipur Hills & 0.000168 \\
\hline J\&K - Jhelum Valley & 2.163806 & Meghalaya & 0.001935 & Haryana - Eastern & 0.000173 \\
\hline Haryana - Eastern & 2.418539 & Manipur Hills & 0.002035 & Meghalaya & 0.000187 \\
\hline Manipur Hills & 2.764995 & J\&K - Mountains & 0.002419 & $\begin{array}{l}\text { Karnataka - } \\
\text { Coastal and Ghats }\end{array}$ & 0.00033 \\
\hline Punjab - Northern & 3.008528 & Punjab - Northern & 0.002673 & Punjab - Northern & 0.000346 \\
\hline Maharashtra - Coastal & 3.024544 & J\&K - Jhelum Valley & 0.002764 & J\&K - Jhelum Valley & 0.000568 \\
\hline Kerala - Southern & 3.660594 & $\begin{array}{l}\text { Karnataka - } \\
\text { Coastal and Ghats }\end{array}$ & 0.003576 & J\&K - Mountains & 0.000622 \\
\hline Meghalaya & 3.845778 & Maharashtra - Coastal & 0.004459 & Maharashtra - Coastal & 0.000945 \\
\hline Punjab - Southern & 4.080785 & Kerala - Southern & 0.00574 & Karnataka - Inland Eastern & 0.001003 \\
\hline Gujarat - Saurashtra & 4.377181 & Himachal Pradesh & 0.007251 & J\&K - Outer Hills & 0.001064 \\
\hline Himachal Pradesh & 5.070846 & $\begin{array}{l}\text { Karnataka - } \\
\text { Inland Eastern }\end{array}$ & 0.007905 & Himachal Pradesh & 0.001445 \\
\hline $\begin{array}{l}\text { Karnataka - } \\
\text { Coastal and Ghats }\end{array}$ & 7.011182 & Gujarat - Saurashtra & 0.008264 & Kerala - Southern & 0.001524 \\
\hline Kerala - Northern & 7.654714 & J\&K - Outer Hills & 0.008611 & $\begin{array}{l}\text { West Bengal - } \\
\text { Central Plains }\end{array}$ & 0.002247 \\
\hline $\begin{array}{l}\text { West Bengal - } \\
\text { Central Plains }\end{array}$ & 7.72978 & Punjab - Southern & 0.008629 & Gujarat - Saurashtra & 0.002419 \\
\hline $\begin{array}{l}\text { Gujarat - } \\
\text { Southern Plains }\end{array}$ & 7.732287 & $\begin{array}{l}\text { West Bengal - Central } \\
\text { Plains }\end{array}$ & 0.010676 & Punjab - Southern & 0.002801 \\
\hline Haryana - Western & 8.988088 & $\begin{array}{l}\text { Maharashtra - } \\
\text { Inland Western }\end{array}$ & 0.015223 & Rajasthan - Northeastern & 0.003237 \\
\hline $\begin{array}{l}\text { Maharashtra - } \\
\text { Inland Western }\end{array}$ & 11.60792 & $\begin{array}{l}\text { Rajasthan - } \\
\text { Northeastern }\end{array}$ & 0.01547 & Assam - Eastern Plains & 0.00335 \\
\hline Rajasthan - Western & 11.98063 & $\begin{array}{l}\text { Assam - } \\
\text { Eastern Plains }\end{array}$ & 0.015635 & $\begin{array}{l}\text { Maharashtra - } \\
\text { Inland Western }\end{array}$ & 0.003752 \\
\hline Assam - Eastern Plains & 11.98233 & Haryana - Western & 0.016983 & Gujarat - Northern Plains & 0.004034 \\
\hline $\begin{array}{l}\text { Rajasthan - } \\
\text { Northeastern }\end{array}$ & 13.21208 & $\begin{array}{l}\text { Gujarat - } \\
\text { Southern Plains }\end{array}$ & 0.017377 & Haryana - Western & 0.004633 \\
\hline $\begin{array}{l}\text { Karnataka - Inland } \\
\text { Eastern }\end{array}$ & 13.41338 & Kerala - Northern & 0.018706 & Rajasthan - Western & 0.004633 \\
\hline Tamilnadu - Inland & 13.66444 & Rajasthan - Western & 0.019114 & Gujarat - Southern Plains & 0.004726 \\
\hline $\begin{array}{l}\text { Karnataka - } \\
\text { Inland Southern }\end{array}$ & 13.79427 & $\begin{array}{l}\text { Gujarat - } \\
\text { Northern Plains }\end{array}$ & 0.020537 & UP - Western & 0.005196 \\
\hline UP - Western & 14.21644 & UP - Western & 0.021512 & $\begin{array}{l}\text { Madhya Pradesh - } \\
\text { Southwestern }\end{array}$ & 0.006241 \\
\hline J\&K - Outer Hills & 14.25788 & $\begin{array}{l}\text { West Bengal - Western } \\
\text { Plains }\end{array}$ & 0.0237 & $\begin{array}{l}\text { West Bengal - Western } \\
\text { Plains }\end{array}$ & 0.006244 \\
\hline Assam - Hills & 15.09375 & Tamilnadu - Inland & 0.023818 & Tamilnadu - Inland & 0.006266 \\
\hline Assam - Western Plains & 15.77768 & Assam - Hills & 0.024916 & Kerala - Northern & 0.006691 \\
\hline $\begin{array}{l}\text { Rajasthan - } \\
\text { Southeastern }\end{array}$ & 15.89191 & $\begin{array}{l}\text { Madhya Pradesh - } \\
\text { Southwestern }\end{array}$ & 0.028624 & Rajasthan - Southern & 0.00675 \\
\hline Tamilnadu - Southern & 16.96555 & $\begin{array}{l}\text { Assam - } \\
\text { Western Plains }\end{array}$ & 0.030199 & Assam - Hills & 0.00763 \\
\hline Gujarat - Northern Plains & 17.15877 & Tamilnadu - Coastal & 0.033359 & Gujarat - Eastern Plains & 0.008515 \\
\hline $\begin{array}{l}\text { West Bengal - } \\
\text { Western Plains }\end{array}$ & 17.19903 & Rajasthan - Southern & 0.033635 & Assam - Western Plains & 0.008648 \\
\hline $\begin{array}{l}\text { Maharashtra - } \\
\text { Inland Northern }\end{array}$ & 17.2416 & Tripura & 0.034107 & Tripura & 0.009084 \\
\hline Tamilnadu - Coastal & 18.57746 & $\begin{array}{l}\text { Maharashtra - Inland } \\
\text { Eastern }\end{array}$ & 0.034448 & $\begin{array}{l}\text { Andhra Pradesh - } \\
\text { Inland Northern }\end{array}$ & 0.009662 \\
\hline $\begin{array}{l}\text { Andhra Pradesh - } \\
\text { Coastal }\end{array}$ & 19.51589 & Tamilnadu - Southern & 0.035141 & $\begin{array}{l}\text { Maharashtra - } \\
\text { Inland Eastern }\end{array}$ & 0.010425 \\
\hline
\end{tabular}




\begin{tabular}{|c|c|c|c|c|c|}
\hline $\begin{array}{l}\text { Maharashtra - Inland } \\
\text { Eastern }\end{array}$ & 19.52682 & $\begin{array}{l}\text { Rajasthan - } \\
\text { Southeastern }\end{array}$ & 0.035786 & Tamilnadu - Coastal & 0.01062 \\
\hline Tripura & 19.58193 & $\begin{array}{l}\text { Maharashtra - } \\
\text { Inland Northern }\end{array}$ & 0.036756 & Tamilnadu - Southern & 0.01062 \\
\hline $\begin{array}{l}\text { Tamilnadu - } \\
\text { Coastal Northern }\end{array}$ & 20.02052 & $\begin{array}{l}\text { Andhra Pradesh - } \\
\text { Coastal }\end{array}$ & 0.037747 & UP - Southern & 0.010719 \\
\hline $\begin{array}{l}\text { Madhya Pradesh - } \\
\text { Southwestern }\end{array}$ & 20.17593 & $\begin{array}{l}\text { Karnataka - } \\
\text { Inland Southern }\end{array}$ & 0.038043 & Andhra Pradesh - Coastal & 0.011133 \\
\hline $\begin{array}{l}\text { West Bengal - } \\
\text { Eastern Plains }\end{array}$ & 20.81617 & $\begin{array}{l}\text { Andhra Pradesh - } \\
\text { Inland Northern }\end{array}$ & 0.038443 & $\begin{array}{l}\text { West Bengal - } \\
\text { Eastern Plains }\end{array}$ & 0.011676 \\
\hline Gujarat - Dry Areas & 22.60888 & UP - Southern & 0.039682 & Gujarat - Dry Areas & 0.012006 \\
\hline $\begin{array}{l}\text { Andhra Pradesh - } \\
\text { Inland Northern }\end{array}$ & 23.36284 & $\begin{array}{l}\text { West Bengal - } \\
\text { Eastern Plains }\end{array}$ & 0.039986 & UP - Central & 0.012565 \\
\hline UP - Central & 23.84277 & $\begin{array}{l}\text { Tamilnadu - } \\
\text { Coastal Northern }\end{array}$ & 0.041806 & Rajasthan - Southeastern & 0.012697 \\
\hline $\begin{array}{l}\text { Madhya Pradesh - } \\
\text { Malwa }\end{array}$ & 24.04948 & Gujarat - Dry Areas & 0.042548 & Madhya Pradesh - Northern & 0.013611 \\
\hline UP - Southern & 24.06105 & $\begin{array}{l}\text { Gujarat - } \\
\text { Eastern Plains }\end{array}$ & 0.043176 & Madhya Pradesh - Malwa & 0.013636 \\
\hline $\begin{array}{l}\text { Andhra Pradesh - Inland } \\
\text { Southern }\end{array}$ & 26.94954 & UP - Central & 0.043939 & West Bengal - Himalayan & 0.013647 \\
\hline Rajasthan - Southern & 27.26148 & $\begin{array}{l}\text { Madhya Pradesh - } \\
\text { Malwa }\end{array}$ & 0.04686 & $\begin{array}{l}\text { Maharashtra - } \\
\text { Inland Northern }\end{array}$ & 0.013807 \\
\hline $\begin{array}{l}\text { West Bengal - } \\
\text { Himalayan }\end{array}$ & 27.92417 & $\begin{array}{l}\text { West Bengal - } \\
\text { Himalayan }\end{array}$ & 0.051908 & Bihar - Northern & 0.014814 \\
\hline Gujarat - Eastern Plains & 28.7604 & Orissa - Coastal & 0.052853 & Orissa - Coastal & 0.014974 \\
\hline $\begin{array}{l}\text { Karnataka - } \\
\text { Inland Northern }\end{array}$ & 29.87136 & $\begin{array}{l}\text { Karnataka - } \\
\text { Inland Northern }\end{array}$ & 0.056475 & $\begin{array}{l}\text { Tamilnadu - } \\
\text { Coastal Northern }\end{array}$ & 0.016148 \\
\hline $\begin{array}{l}\text { Maharashtra - } \\
\text { Inland Central }\end{array}$ & 29.94484 & $\begin{array}{l}\text { Maharashtra - } \\
\text { Inland Central }\end{array}$ & 0.058231 & Bihar - Central & 0.016644 \\
\hline Orissa - Coastal & 30.81099 & $\begin{array}{l}\text { Madhya Pradesh - } \\
\text { Northern }\end{array}$ & 0.058277 & Karnataka - Inland Southern & 0.016813 \\
\hline Maharashtra - Eastern & 31.64293 & Bihar - Central & 0.059304 & $\begin{array}{l}\text { Maharashtra - Inland } \\
\text { Central }\end{array}$ & 0.017019 \\
\hline $\begin{array}{l}\text { Madhya Pradesh - } \\
\text { Vindhya }\end{array}$ & 31.71062 & Bihar - Northern & 0.059516 & Karnataka - Inland Northern & 0.017207 \\
\hline Bihar - Central & 32.00861 & $\begin{array}{l}\text { Madhya Pradesh - } \\
\text { Vindhya }\end{array}$ & 0.064609 & Madhya Pradesh - Vindhya & 0.019062 \\
\hline $\begin{array}{l}\text { Madhya Pradesh - } \\
\text { Northern }\end{array}$ & 34.25524 & $\begin{array}{l}\text { Andhra Pradesh - } \\
\text { Inland Southern }\end{array}$ & 0.06763 & UP - Eastern & 0.021517 \\
\hline Bihar - Northern & 34.29257 & UP - Eastern & 0.073751 & Madhya Pradesh - Central & 0.022072 \\
\hline $\begin{array}{l}\text { Madhya Pradesh - } \\
\text { Central }\end{array}$ & 35.46721 & $\begin{array}{l}\text { Madhya Pradesh - } \\
\text { Central }\end{array}$ & 0.074836 & $\begin{array}{l}\text { Andhra Pradesh - } \\
\text { Inland Southern }\end{array}$ & 0.023824 \\
\hline UP - Eastern & 36.27837 & Maharashtra - Eastern & 0.078661 & Maharashtra - Eastern & 0.024436 \\
\hline Madhya Pradesh - South & 41.44052 & $\begin{array}{l}\text { Madhya Pradesh - } \\
\text { South }\end{array}$ & 0.097987 & Madhya Pradesh - South & 0.031327 \\
\hline Orissa - Northern & 45.7791 & Orissa - Northern & 0.117399 & Orissa - Northern & 0.043054 \\
\hline $\begin{array}{l}\text { Andhra Pradesh - } \\
\text { Southwestern }\end{array}$ & 54.46258 & $\begin{array}{l}\text { Andhra Pradesh - } \\
\text { Southwestern }\end{array}$ & 0.16089 & Orissa - Southern & 0.061833 \\
\hline Orissa - Southern & 55.68906 & Orissa - Southern & 0.165496 & $\begin{array}{l}\text { Andhra Pradesh - } \\
\text { Southwestern }\end{array}$ & 0.062971 \\
\hline
\end{tabular}

In Table 10 we report on the Gini coefficients in these regions again arranged in ascending order. 


\section{Table 10: Gini Coefficients in NSS regions}

\begin{tabular}{|c|c|c|c|c|c|}
\hline \multicolumn{2}{|l|}{ 30-day recall } & \multicolumn{2}{|l|}{ 7-day recall } & \multicolumn{2}{|l|}{ Combined data } \\
\hline Manipur Hills & 0.11239 & Manipur Hills & 0.11079 & Manipur Hills & 0.11188 \\
\hline Manipur Plains & 0.18049 & J\&K - Outer Hills & 0.13973 & Manipur Plains & 0.17562 \\
\hline J\&K - Jhelum Valley & 0.21909 & Assam - Hills & 0.16327 & Assam - Hills & 0.19526 \\
\hline Assam - Hills & 0.22145 & Manipur Plains & 0.16933 & J\&K - Jhelum Valley & 0.21503 \\
\hline J\&K - Outer Hills & 0.24019 & $\begin{array}{l}\text { Madhya Pradesh - } \\
\text { Southwestern }\end{array}$ & 0.18242 & $\begin{array}{l}\text { Madhya Pradesh - } \\
\text { Southwestern }\end{array}$ & 0.21943 \\
\hline Bihar - Northern & 0.24126 & Meghalaya & 0.21329 & J\&K - Outer Hills & 0.22000 \\
\hline $\begin{array}{l}\text { Karnataka - Coastal and } \\
\text { Ghats }\end{array}$ & 0.24388 & J\&K - Jhelum Valley & 0.21625 & Meghalaya & 0.22983 \\
\hline Meghalaya & 0.24404 & Karnataka - Inland Eastern & 0.22444 & $\begin{array}{l}\text { Karnataka - } \\
\text { Coastal and Ghats }\end{array}$ & 0.24522 \\
\hline Rajasthan - Southeastern & 0.24477 & Tripura & 0.24152 & Bihar - Northern & 0.24859 \\
\hline $\begin{array}{l}\text { Madhya Pradesh - } \\
\text { Southestern }\end{array}$ & 0.24609 & Karnataka - Coastal and Ghats & 0.24563 & Tripura & 0.25180 \\
\hline Madhya Pradesh - Vindhya & 0.24971 & Bihar - Northern & 0.24883 & UP - Southern & 0.25236 \\
\hline UP - Southern & 0.2508 & Assam - Western Plains & 0.24883 & $\begin{array}{l}\text { Karnataka - } \\
\text { Inland Eastern }\end{array}$ & 0.25381 \\
\hline Punjab - Northern & 0.25649 & Rajasthan - Southern & 0.24919 & Haryana - Western & 0.25941 \\
\hline Tripura & 0.25951 & Haryana - Western & 0.25187 & Assam - Western Plains & 0.26137 \\
\hline Rajasthan - Western & 0.26048 & UP - Southern & 0.25449 & Madhya Pradesh - Vindhya & 0.26400 \\
\hline Assam - Western Plains & 0.26312 & Maharashtra - Inland Northern & 0.25478 & Assam - Eastern Plains & 0.26468 \\
\hline Assam - Eastern Plains & 0.26317 & Madhya Pradesh - Northern & 0.25747 & Rajasthan - Southern & 0.26665 \\
\hline Karnataka - Inland Eastern & 0.26547 & Assam - Eastern Plains & 0.25809 & Gujarat - Saurashtra & 0.27066 \\
\hline Haryana - Western & 0.26674 & Karnataka - Inland Northern & 0.26799 & $\begin{array}{l}\text { Maharashtra - } \\
\text { Inland Northern }\end{array}$ & 0.27128 \\
\hline Gujarat - Saurashtra & 0.26899 & Madhya Pradesh - Vindhya & 0.27128 & Rajasthan - Western & 0.27569 \\
\hline Maharashtra - Eastern & 0.27177 & Gujarat - Saurashtra & 0.27146 & $\begin{array}{l}\text { Karnataka - } \\
\text { Inland Northern }\end{array}$ & 0.27956 \\
\hline Maharashtra - Inland Central & 0.27182 & West Bengal - Eastern Plains & 0.27642 & Madhya Pradesh - Northern & 0.28368 \\
\hline Orissa - Coastal & 0.27493 & Haryana - Eastern & 0.28495 & $\begin{array}{l}\text { West Bengal - } \\
\text { Eastern Plains }\end{array}$ & 0.28490 \\
\hline Rajasthan - Southern & 0.2754 & West Bengal - Western Plains & 0.28576 & $\begin{array}{l}\text { West Bengal - } \\
\text { Western Plains }\end{array}$ & 0.28818 \\
\hline $\begin{array}{l}\text { West Bengal - Western } \\
\text { Plains }\end{array}$ & 0.27837 & Rajasthan - Western & 0.28983 & Haryana - Eastern & 0.29034 \\
\hline $\begin{array}{l}\text { Maharashtra - Inland } \\
\text { Northern }\end{array}$ & 0.28147 & Gujarat - Dry Areas & 0.29377 & Orissa - Coastal & 0.29182 \\
\hline West Bengal - Eastern Plains & 0.28278 & Bihar - Central & 0.29509 & Gujarat - Dry Areas & 0.29385 \\
\hline Karnataka - Inland Northern & 0.28706 & J\&K - Mountains & 0.29654 & Punjab - Northern & 0.29601 \\
\hline Maharashtra - Inland Eastern & 0.28843 & Orissa - Coastal & 0.29759 & J\&K - Mountains & 0.30041 \\
\hline Haryana - Eastern & 0.29132 & Gujarat - Eastern Plains & 0.30148 & Gujarat - Eastern Plains & 0.30251 \\
\hline Gujarat - Dry Areas & 0.29428 & Rajasthan - Northeastern & 0.3029 & Bihar - Central & 0.30484 \\
\hline Tamilnadu - Southern & 0.29634 & $\begin{array}{l}\text { Andhra Pradesh - } \\
\text { Inland Southern }\end{array}$ & 0.31056 & Rajasthan - Northeastern & 0.31143 \\
\hline Madhya Pradesh - Central & 0.29698 & Kerala - Northern & 0.3109 & UP - Eastern & 0.31147 \\
\hline Madhya Pradesh - Northern & 0.29727 & Andhra Pradesh - Coastal & 0.31293 & Maharashtra - Eastern & 0.31184 \\
\hline Madhya Pradesh - Malwa & 0.3002 & Gujarat - Northern Plains & 0.31535 & $\begin{array}{l}\text { Maharashtra - } \\
\text { Inland Central }\end{array}$ & 0.31319 \\
\hline
\end{tabular}




\begin{tabular}{|c|c|c|c|c|c|}
\hline Gujarat - Eastern Plains & 0.30186 & UP - Eastern & 0.31865 & Madhya Pradesh - Central & 0.31381 \\
\hline Madhya Pradesh - South & 0.30562 & Punjab - Northern & 0.31891 & $\begin{array}{l}\text { Maharashtra - } \\
\text { Inland Eastern }\end{array}$ & 0.31758 \\
\hline UP - Eastern & 0.31145 & Madhya Pradesh - Central & 0.32011 & $\begin{array}{l}\text { Andhra Pradesh - } \\
\text { Inland Southern }\end{array}$ & 0.32031 \\
\hline J\&K - Mountains & 0.31474 & West Bengal - Himalayan & 0.32852 & Rajasthan - Southeastern & 0.32448 \\
\hline Bihar - Central & 0.31493 & Orissa - Southern & 0.33006 & Orissa - Southern & 0.32587 \\
\hline $\begin{array}{l}\text { Andhra Pradesh - Inland } \\
\text { Southern }\end{array}$ & 0.32049 & UP - Western & 0.33035 & Andhra Pradesh - Coastal & 0.32817 \\
\hline Rajasthan - Northeastern & 0.32232 & Tamilnadu - Coastal & 0.33114 & Madhya Pradesh - Malwa & 0.32891 \\
\hline Himachal Pradesh & 0.32247 & Maharashtra - Eastern & 0.33337 & West Bengal - Himalayan & 0.32912 \\
\hline Gujarat - Southern Plains & 0.32535 & Tamilnadu - Inland & 0.34077 & Gujarat - Northern Plains & 0.33215 \\
\hline Orissa - Southern & 0.32565 & Himachal Pradesh & 0.34237 & Kerala - Northern & 0.33545 \\
\hline West Bengal - Himalayan & 0.33393 & Maharashtra - Inland Central & 0.34323 & Himachal Pradesh & 0.33768 \\
\hline Andhra Pradesh - Coastal & 0.33528 & Maharashtra - Inland Eastern & 0.344 & Tamilnadu - Southern & 0.33839 \\
\hline Maharashtra - Coastal & 0.3427 & Kerala - Southern & 0.34728 & Tamilnadu - Coastal & 0.34008 \\
\hline Gujarat - Northern Plains & 0.34333 & Karnataka - Inland Southern & 0.34882 & UP - Western & 0.34064 \\
\hline UP - Western & 0.34413 & Madhya Pradesh - Malwa & 0.35481 & Tamilnadu - Inland & 0.34991 \\
\hline Tamilnadu - Coastal & 0.34481 & Maharashtra - Inland Western & 0.36339 & Madhya Pradesh - South & 0.36045 \\
\hline Punjab - Southern & 0.34986 & Tamilnadu - Southern & 0.36547 & $\begin{array}{l}\text { Maharashtra - } \\
\text { Inland Western }\end{array}$ & 0.36302 \\
\hline Tamilnadu - Inland & 0.35354 & Madhya Pradesh - South & 0.36839 & Kerala - Southern & 0.36796 \\
\hline $\begin{array}{l}\text { Maharashtra - } \\
\text { Inland Western }\end{array}$ & 0.36217 & Andhra Pradesh - Southwestern & 0.37559 & Punjab - Southern & 0.36863 \\
\hline UP - Central & 0.36428 & Punjab - Southern & 0.37882 & Gujarat - Southern Plains & 0.37279 \\
\hline Kerala - Northern & 0.36466 & Rajasthan - Southeastern & 0.38046 & $\begin{array}{l}\text { Karnataka - } \\
\text { Inland Southern }\end{array}$ & 0.37363 \\
\hline Orissa - Northern & 0.3655 & Maharashtra - Coastal & 0.38954 & UP - Central & 0.37705 \\
\hline $\begin{array}{l}\text { Andhra Pradesh - } \\
\text { South Western }\end{array}$ & 0.37606 & UP - Central & 0.39098 & Maharashtra - Coastal & 0.38067 \\
\hline West Bengal - Central Plains & 0.37623 & $\begin{array}{l}\text { Andhra Pradesh - } \\
\text { Inland Northern }\end{array}$ & 0.39856 & $\begin{array}{l}\text { Andhra Pradesh - } \\
\text { Southwestern }\end{array}$ & 0.38193 \\
\hline Kerala - Southern & 0.38705 & Gujarat - Southern Plains & 0.40994 & Orissa - Northern & 0.4015 \\
\hline Karnataka - Inland Southern & 0.38898 & Orissa - Northern & 0.42921 & $\begin{array}{l}\text { West Bengal - } \\
\text { Central Plains }\end{array}$ & 0.40729 \\
\hline $\begin{array}{l}\text { Andhra Pradesh - } \\
\text { Inland Northern }\end{array}$ & 0.43951 & West Bengal - Central Plains & 0.43178 & $\begin{array}{l}\text { Andhra Pradesh - } \\
\text { Inland Northern }\end{array}$ & 0.42283 \\
\hline Tamilnadu - Coastal Northern & 0.4628 & Tamilnadu - Coastal Northern & 0.47837 & $\begin{array}{l}\text { Tamilnadu - } \\
\text { Coastal Northern }\end{array}$ & 0.47036 \\
\hline
\end{tabular}

We explore patterns of regional inequality in respect of mean consumption, the three FGT measures of poverty and inequality in table 11 (for variation across states) and table 12 (for variation across the 63 NSS regions). 
Table 11: Variation of Mean Consumption, Poverty and Inequality across Indian States

\begin{tabular}{|l|l|l|c|c|c|}
\hline & & Average & Highest & Lowest & $\begin{array}{c}\text { Coefficient of } \\
\text { Variation }\end{array}$ \\
\hline \multirow{3}{*}{$\begin{array}{l}\text { Mean Consumption } \\
\text { (Rs. per capita per } \\
\text { month) }\end{array}$} & 30 day recall & 802.64 & 1220.35 & 507.98 & 0.24 \\
\cline { 2 - 6 } & 7 day recall & 865.32 & 1267.08 & 546.15 & 0.237 \\
\cline { 2 - 6 } & Combined & 832.49 & 1242.27 & 531.10 & 0.238 \\
\hline \multirow{4}{*}{ PG0 } & 30 day recall & 18.25 & 48.97 & 1.43 & 0.70 \\
\cline { 2 - 6 } & 7 day recall & 13.53 & 34.07 & 0.348 & 0.73 \\
\cline { 2 - 6 } & Combined & 15.96 & 41.76 & 0.92 & 0.71 \\
\hline \multirow{5}{*}{ PG1 } & 30 day recall & 0.035 & 0.121 & 0.001 & 0.84 \\
\cline { 2 - 6 } & 7 day recall & 0.02 & 0.075 & 0.0002 & 0.89 \\
\cline { 2 - 6 } & Combined & 0.029 & 0.0989 & 0.000682 & 0.85 \\
\hline \multirow{3}{*}{ Gini Coefficient } & 30 day recall & 0.0107 & 0.042 & $9.41 \mathrm{E}-05$ & 0.95 \\
\cline { 2 - 6 } & 7 day recall & 0.0069 & 0.025 & $1.34 \mathrm{E}-05$ & 0.995 \\
\cline { 2 - 6 } & Combined & 0.0088 & 0.0344 & $5.26 \mathrm{E}-05$ & 0.958 \\
\cline { 2 - 6 } & 30 day recall & 0.314 & 0.398 & 0.197 & 0.171 \\
\cline { 2 - 6 } & 7 day recall & 0.315 & 0.405 & 0.189 & 0.190 \\
\cline { 2 - 6 } & Combined & 0.317 & 0.402 & 0.193 & 0.1778 \\
\hline
\end{tabular}

It is interesting to note that the 7-day recall magnitudes are generally more volatile than 30 day magnitudes with the combined results lying in between. Further, the coefficients of variation of the FGT measures are much higher than those for mean consumption and the Gini coefficient. Within the FGT measures PG2 has higher coefficient of variation than PG1 which in turn has a higher coefficient of variation than PG0. Thus poverty, particularly severe poverty, is more volatile.

Table 12: Variation of Mean Consumption, Poverty and Inequality across 63 NSS regions

\begin{tabular}{|l|l|c|c|c|c|}
\hline & & Average & Highest & Lowest & $\begin{array}{c}\text { Coefficient of } \\
\text { Variation }\end{array}$ \\
\hline \multirow{3}{*}{$\begin{array}{l}\text { Mean Consumption } \\
\text { Rs. per capita per } \\
\text { month) }\end{array}$} & 30 day recall & 758.91 & 1504.58 & 382.32 & 0.304 \\
\hline \multirow{4}{*}{ PG0 } & 7 day recall & 822.57 & 1677.62 & 521.35 & 0.294 \\
\cline { 2 - 6 } & Combined & 788.91 & 1614.20 & 435.02 & 0.300 \\
\hline \multirow{4}{*}{ PG1 } & 30 day recall & 21.84 & 71.98 & 0 & 0.668 \\
\cline { 2 - 6 } & 7 day recall & 16.219 & 45.428 & 0 & 0.964 \\
\cline { 2 - 6 } & Combined & 19.03 & 55.689 & 0 & 0.675 \\
\hline \multirow{5}{*}{ PG2 } & 30 day recall & 0.043 & 0.2278 & 0 & 0.945 \\
\cline { 2 - 6 } & 7 day recall & 0.03 & 0.124 & 0 & 0.964 \\
\cline { 2 - 6 } & Combined & 0.036 & 0.165 & 0 & 0.923 \\
\hline \multirow{3}{*}{ Gini Coefficient } & 30 day recall & 0.013 & 0.087 & 0 & 1.131 \\
\cline { 2 - 6 } & 7 day recall & 0.008 & 0.045 & 0 & 0.964 \\
\cline { 2 - 6 } & Combined & 0.011 & 0.062 & 0 & 1.117 \\
\hline
\end{tabular}


We offer additional comments by concentrating on the upper and lower tails of the distributions for average, consumption, FGT indices and the consumption Ginis. In order to avoid tedious comparisons, we will first pick the lowest 5 regions in terms of per capita consumption and the highest five, using the 30-day recall. How many of these overlap with those with the lowest or highest FGT indices and Gini coefficients would help understand better spatial dimensions of well-being, deprivation and inequality.

I. The regions with the lowest per capita expenditure (in ascending order) $\operatorname{are}^{10}$ :

Orissa-Southern

Bihar-Northern

Andhra Pradesh-South western

Madhya Pradesh-South

Orissa-Coastal.

Those with the highest per capita expenditure (in ascending order) are:

Tamil Nadu-Coastal Northern

Punjab-Southern

J\&K-Mountains

Kerala-Southern

Maharashtra-Coastal

II. It turns out that three out of the five regions with the lowest per capita expenditure are also the ones with the highest incidence of poverty (PG0). These are (in ascending order):

Madhya Pradesh-South

Andhra Pradesh-South Western

Orissa-Southern

${ }^{10}$ Manipur Hills is omitted, as it belongs to a relatively small state. 
Let us now examine the overlap with regions with the highest distributionally sensitive poverty (PG2). It turns out that the regions with the highest incidence of poverty are also the ones with the highest PG2.

III. There is some overlap between regions with the highest per capita expenditure and lowest incidence of poverty (PG0). These are :

J\&K-Mountains

Maharashtra-Coastal

The overlap with regions with the lowest PG2 is smaller, as it comprises just one region viz. Maharashtra -Coastal.

IV. Let us now turn to the overlap between lowest consumption and lowest Gini. The overlap is small comprising Madhya Pradesh-South.

V. The overlap between regions with the highest consumption and Gini is not so small, as it comprises Kerala-Southern, and Tamil Nadu-Coastal Northern.

VI.Finally, let us examine the overlap between the Ginis and FGT indices. The regions with the highest Ginis (in ascending order) are:

West Bengal-Central Plains

Kerala-Southern

Karnataka-Inland Southern

Andhra Pradesh-Inland Northern

Tamil Nadu-Coastal Northern.

None of these regions overlaps with those with the highest incidence of poverty (PG0). However, there is some overlap with regions with the highest distributionally sensitive poverty, comprising Karnataka -Inland Southern. 


\section{Concluding Remarks}

Even though we have not gone beyond ordinal comparisons with the estimates of poverty and inequality from the previous $55^{\text {th }}$ round of the NSS, some of the findings are noteworthy. First, more than a few of the states (e.g. UP, Madhya Pradesh, Orissa), with high levels of poverty measured using the FGT class of poverty indices, and high concentrations of the poor in rural areas continue to do so in the $60^{\text {th }}$ round of the NSS. Second, except for the small north eastern states, high levels of inequality in consumption expenditure distribution persisted in many of the states. Third, the overlap between regions with the lowest consumption expenditure and highest poverty, and between those with highest consumption and lowest poverty was far from negligible. Fourth, the overlaps between lowest consumption expenditure and lowest Ginis, and between highest consumption and highest Ginis were negligible or small. Fifth, in general, the 7-day recall magnitudes (average consumption expenditure, poverty and inequality) are more volatile than 30-day magnitudes. Sixth, the CVs of the FGT measures are higher than those for mean consumption and the Gini coefficient. Finally, the CVs of PG2 (or distributionally sensitive poverty) are higher than those of PG1 (intensity of poverty) which in turn are higher than those of PG0 (or the head-count

ratio). It is surmised that despite impressive growth rates deprivation is pervasive, pockets of severe poverty persist, and inequality is rampant. 


\section{References}

Datt, G., V. Kozel and M. Ravallion (2005) “A Model-Based Assessment of India’s Progress in Reducing Poverty in the 1990s”, in Deaton and Kozel (eds).

Deaton, A. “Adjusted Indian Poverty Estimates for 1999/2000”, in Deaton and Kozel (eds).

Deaton, A. and V. Kozel (eds) (2005) The Great Indian Poverty Debate, New Delhi: Macmillan.

Gaiha, R. (1995) “Does Agricultural Growth Matter in Poverty Alleviation?’, Development and Change, 26(2): 285-304.

Gaiha, R., and K. Imai (2004) "Vulnerability, Shocks and Persistent of Poverty: Estimates for Semi-Arid Rural South India”, Oxford Development Studies, 32(2): 261-81.

Gaiha, R. and V. Kulkarni (2005) “Anthropometric Failure and Persistence of Poverty in Rural India”, International Review of Applied Economics, 19(2): 179-97.

Gaiha, R., K. Imai and M.A. Nandhi (2006) "Millennium Development Goal of Halving Poverty in Asia and the Pacific Region — Progress, Prospects and Priorities”, Rome: IFAD, Occasional Papers 1.

Jha, R. and R. Gaiha (2003) "The Determinants of Undernutrition in Rural India”, ASARC Working Paper, 2003/01, Australian National University.

Jha, R. and A. Sharma (2003) "Spatial Distribution of Rural Poverty - Last Three Quinquennial Rounds of the NSS” Economic and Political Weekly, 39: 4985-93 (22 November).

Kijima, Y. and P. Lanjouw (2003) "Poverty in India During the 1990s: A Regional Perspective”, Washington DC: World Bank, World Bank Policy Research Working Paper 3141.

Nayyar, R. (2003) “India Country Study”, New Delhi: Planning Commission, Government of India (mimeo).

Sen, A. and Himanshu (2004) "Poverty and Inequality in India-I”, Economic and Political Weekly, 40: 4247-63 (18 September).

Sen, A, and Himanshu (2005) "Poverty and Inequality in India”, in Deaton and Kozel (eds).

Sundaram, K. and S. Tendulkar (2005) "Poverty Outcomes in India in the 1990s”, in Deaton and Kozel (eds).

Sundaram, K. and S. Tendulkar (2003) "Poverty in India in the 1990s - Revised Results for All-India and 15 Major States for 1993-94”, Economic and Political Weekly, 39:

4826-72 (15 November). 